\title{
SF-DEM: a new instrument for assessing social functioning in dementia
}

Andrew Sommerlad, David Singleton, Rebecca Jones, Sube Banerjee, Gill Livingston

\section{Poster 87}

University College London, London, UK (A Sommerlad MSc, D Singleton MSc, R Jones MSc, G Livingston MD); and Brighton and Sussex Medical School, Brighton, UK (S Banerjee MD)

\section{Correspondence to:}

Dr Andrew Sommerlad, University College London, Maple House, London W1T 7NF, UK

a.sommerlad@ucl.ac.uk

\begin{abstract}
Background There is no standardised instrument for assessing social functioning in dementia, even though decline in social functioning is one of the diagnostic criteria for dementia and important to patients and their families. We aimed to develop a valid, reliable, acceptable instrument for assessing social function in people with dementia.
\end{abstract}

Methods We conducted qualitative interviews with 18 dyads of people with dementia and their family carers, a literature review, and focus groups with expert health-care professionals to develop the Social Functioning in Dementia (SF-DEM) instrument. SF-DEM measures the social functioning of a person with dementia using their own rating or a family carer's rating. We tested acceptability and psychometric properties of these measures in structured interviews at baseline and at 4 weeks' and 6-8 months' follow-up in a cohort of 30 dyads of people with mild dementia and their carers.

Findings SF-DEM had content validity. The instrument was acceptable to both patients and carers, who all rated it as acceptable or very acceptable. Inter-rater agreement was good or very good for all questions. Test-retest reliability was very strong for the carer-rated SF-DEM (intraclass correlation coefficient $0.89,95 \% \mathrm{Cl} 0.73$ to 0.96$)$ and patient-rated version $(0.80,0.54$ to 0.92$)$, and both versions had internal consistency (Cronbach's $\alpha=0.71$ for carer-rated SF-DEM and 0.64 for patient-rated). SF-DEM had concurrent validity, since it was moderately correlated with a question about overall social functioning $(r=0.60,95 \% \mathrm{Cl} 0.29$ to 0.78 for carer-rated; $0.44,0.07$ to 0.68 for patient-rated). SF-DEM also had convergent validity, as evidenced by a moderate correlation between patient and carer ratings $(r=0 \cdot 59,95 \% \mathrm{Cl} 0.07$ to $0 \cdot 81)$. At follow-up (mean duration $7 \cdot 2$ months, SD 0.5)), patient-rated SF-DEM score increased by 1.3 points $(95 \% \mathrm{Cl}-0.3$ to $2 \cdot 9, \mathrm{p}=0.10)$ and caregiver-rated SF-DEM score increased by 1.4 points $(-0.1$ to $2 \cdot 9, p=0.06)$ for each point on a five point ordinal scale of social change. 
Interpretation Patient-rated and carer-rated versions of the SF-DEM are reliable, valid, and acceptable measures of social function in people with mild dementia. Further research is required to test the generalisability to other populations.

Funding None.

\section{Contributors}

AS and GL conceptualised the study, and were responsible for methodology. AS drafted the abstract. $A S$ and $D S$ conducted the investigation. $A S$ and $R B$ analysed the data. $A S, R J, S B$, and GL reviewed and edited the abstract. GL supervised the study.

\section{Declaration of interests}

We declare no competing interests. 